\title{
Analysis of a Dengue Disease Model with Nonlinear Incidence
}

\author{
Shu-Min Guo, ${ }^{1}$ Xue-Zhi Li, ${ }^{2}$ and Mini Ghosh ${ }^{3}$ \\ ${ }^{1}$ Department of Mathematics and Information Science, Shaoguan University, Shaoguan 512005, China \\ ${ }^{2}$ Department of Mathematics, Xinyang Normal University, Xinyang 464000, China \\ ${ }^{3}$ School of Advanced Sciences, VIT University, Chennai Campus, Chennai 600048, India \\ Correspondence should be addressed to Xue-Zhi Li; xzli66@126.com
}

Received 10 September 2012; Revised 3 January 2013; Accepted 3 January 2013

Academic Editor: Eric Campos Canton

Copyright (c) 2013 Shu-Min Guo et al. This is an open access article distributed under the Creative Commons Attribution License, which permits unrestricted use, distribution, and reproduction in any medium, provided the original work is properly cited.

A dengue disease epidemic model with nonlinear incidence is formulated and analyzed. The equilibria and threshold of the model are found. The stability of the system is analyzed through a geometric approach to stability. The proposed model also exhibits backward bifurcation under suitable conditions on parameters. Our results imply that a nonlinear incidence produces rich dynamics and they should be studied carefully in order to analyze the spread of disease more accurately. Finally, numerical simulations are presented to illustrate the analytical findings.

\section{Introduction}

Dengue disease is transmitted in humans by mosquitoes, called vectors, who carry the disease without getting it themselves. Every year many people die from dengue disease. It has become a global health problem due to its rapid spread. In order to predict the development extent of such epidemics, it is useful to find the suitable mathematical models, which can provide insight into the dynamics of a disease and can help us to make right decisions on public health policies.

However in the region of high infective prevalence such as Africa, some tropical countries, and so forth, there are lots of people infected with dengue, and so forth. and hence there are lots of infected mosquitoes. So in these endemic regions residents are frequently bitten by infected mosquitoes, thus making individuals exposed with multiple bites of mosquito which causes individuals to move to infected class with increased rate compared to people who are exposed with single effective bite of mosquito. So it is reasonable to consider nonlinear saturation incidence in place of bilinear incidence or standard incidence in the model as it also incorporates the effects of increased exposure to mosquito bites.

In fact the incidence rate is very important in the transmission dynamics of the disease and the qualitative behaviour of the disease changes with the change in the incidence rate.
In a recent paper by Xiao and Tang [1], interaction of the nonlinear incidence and the partial immunity for a simple SIV epidemic model is investigated and it is shown that due to the nonlinear incidence, vaccination may contribute to disease spread rather than to its elimination. A vector-host epidemic model with nonlinear incidence is also analyzed by Cai and $\mathrm{Li}$ [2]. Here they considered nonlinear saturating type incidence and they studied the effect of this type of incidence rate on the basic reproduction number and the equilibrium level of the infective population. Hu et al. [3] analyzed an SIR epidemic model with nonlinear incidence rate and treatment. Here it is shown that reducing the basic reproduction number below one is not enough to eliminate the disease as backward bifurcation occurs for this model. Also when the basic reproduction number is greater than unity, there are multiple endemic equilibria. The conditions for the stability of endemic equilibrium and the existence of limit cycle are discussed in this paper. A discrete-time epidemic model with nonlinear incidence rates is studied by $\mathrm{Li}$ et al. [4], where a very complex dynamical behaviors are demonstrated. It is shown that there are possibilities of the transcritical bifurcation, flip bifurcation, Hopf bifurcation and chaos. A vector-borne model with nonlinear incidence is analyzed by Ozair et al. [5]. Here they considered the conditions for the stability of endemic equilibrium. 
In recent years, dengue has become a major public health problem and is endemic in several countries. It is affecting almost one-third of the world's population. Dengue is an infection caused by a virus known as flavivirus and is spread by their vector Aedes aegypti. There are four different serotypes of flavivirus but infection with one serotype gives life-long immunity to that serotype but not to other three serotypes. So one can formulate dengue disease model as SIS or SIR model depending upon serotypes of flavivirus. In this paper we have formulated an SIR epidemic model with nonlinear incidence assuming the infection of dengue is caused by the flavivirus which is giving life-long immunity. In dengue-endemic area a person can be infected with more than one serotype of flavivirus in his/her lifetime. But in the current study we have ignored this fact as it will complicate the model and its analysis.

The paper is organized as follows. In Section 2 the model and some preliminary properties are presented. In Section 3 equilibria and bifurcation are studied. Section 4 is devoted to the global stability analysis of the endemic equilibrium, when it is unique. Numerical simulations are given in Section 5, we end the paper with conclusions in Section 6.

\section{Model Formulation}

The vector-host epidemic model with nonlinear incidence can be described by the following system of differential equations:

$$
\begin{gathered}
\frac{d S(t)}{d t}=b_{1}-\lambda_{1} S(t) V(t)[1+\alpha V(t)]-\mu_{1} S(t), \\
\frac{d I(t)}{d t}=\lambda_{1} S(t) V(t)[1+\alpha V(t)]-\gamma I(t)-\mu_{1} I(t), \\
\frac{d R(t)}{d t}=\gamma I(t)-\mu_{1} R(t), \\
\frac{d M(t)}{d t}=b_{2}-\lambda_{2} M(t) I(t)-\mu_{2} M(t), \\
\frac{d V(t)}{d t}=\lambda_{2} M(t) I(t)-\mu_{2} V(t) .
\end{gathered}
$$

The total host population size at time $t$, given by $N_{1}(t)$, is partitioned into subclasses of individuals who are susceptible, infectious and recovered, with sizes denoted by $S(t), I(t)$ and $R(t)$, respectively. Furthermore, the host population dies at a natural death rate $\mu_{1}$. In addition, the host population is recruited at a rate $b_{1}$. We assume that vertical transmission in the host does not occur so that all newly recruited individuals are susceptible. The per capita recovery rate of the hosts is given by $\gamma$. The recovered individuals are assumed to acquire permanent immunity and there is no transfer from the $R$ class back to the $S$ class. The nonlinear incidence rate is $\lambda_{1} S(t) V(t)[1+\alpha V(t)]$, where $\lambda_{1}$ and $\alpha$ are positive constants, $V(t)$ is the number of vectors at time $t$ who carry the pathogen. It corresponds to an increased rate of infection due to two exposures over a short time period. The single contacts lead to infection at the rate $\lambda_{1} S(t) V(t)$, whereas the new infective individuals arise from double exposures at a rate $\alpha \lambda_{1} S(t) V^{2}(t)$.

The second component of the vector population is the number of pathogen-free (susceptible) vectors at time $t$, given by $M(t)$. The total size of the vector population at time $t$, given by $N_{2}(t)$ and is subdivided into these two vector-population classes, the susceptible vectors and infectious vectors. The vector population dies at a natural death rate $\mu_{2}$. In addition, the vector population is recruited at a birth rate $b_{2}$. Although there are evidences that the pathogen of several vectorborne diseases (e.g., West Nile fever, yellow fever and Lyme disease) can be transmitted from (female) parent to offspring in the vector population, we will assume that all newborn vectors are susceptible and vertical transmission is neglected. Susceptible vectors start carrying the pathogen after getting into contact (biting) an infective host at a rate $\lambda_{2}$ so that the incidence of newly infected vectors is given by a mass-action term $\lambda_{2} M(t) I(t)$. In contrast to the host population, once the vectors become carriers of the microparasite, they carry it for life.

We make some reasonable technical assumptions on the parameters of the model, namely that $\gamma>0, \mu_{j}>0$ and $b_{j}>0$ for $j=1,2$. The above systems for the host population and the vector are also equipped with initial conditions as follows: $S(0)=S_{0}, I(0)=I_{0}, R(0)=\bar{R}_{0}, M(0)=M_{0}$ and $V(0)=V_{0}$.

The total population size $N_{1}(t)$ can be determined by $N_{1}(t)=S(t)+I(t)+R(t)$ or from the differential equation

$$
\frac{d N_{1}(t)}{d t}=b_{1}-\mu_{1} N_{1}(t)
$$

which is derived by adding the first three equations in (1). The total number of vectors $N_{2}(t)$ can be determined by $N_{2}(t)=$ $M(t)+V(t)$ or from the differential equation

$$
\frac{d N_{2}(t)}{d t}=b_{2}-\mu_{2} N_{2}
$$

which is derived by adding the last two equations in (1).

It is easily seen that both for the host population and for the vector population the corresponding total population sizes are asymptotically constant:

$$
\lim _{t \rightarrow \infty} N_{1}(t)=\frac{b_{1}}{\mu_{1}}, \quad \lim _{t \rightarrow \infty} N_{2}(t)=\frac{b_{2}}{\mu_{2}} .
$$

So we can assume without loss of generality that $N_{1}(t)=$ $b_{1} / \mu_{1}, N_{2}(t)=b_{2} / \mu_{2}$ for all $t \geq 0$.

Then the dynamics of system (1) is qualitatively equivalent to the dynamics of system given by

$$
\begin{gathered}
\frac{d S(t)}{d t}=b_{1}-\lambda_{1} S(t) V(t)[1+\alpha V(t)]-\mu_{1} S(t), \\
\frac{d I(t)}{d t}=\lambda_{1} S(t) V(t)[1+\alpha V(t)]-\gamma I(t)-\mu_{1} I(t), \\
\frac{d V(t)}{d t}=\lambda_{2}\left[\frac{b_{2}}{\mu_{2}}-V(t)\right] I(t)-\mu_{2} V(t) .
\end{gathered}
$$

The values of $R$ and $M$ can be determined correspondingly from $R=b_{1} / \mu_{1}-S-I$, and $M=b_{2} / \mu_{2}-V$ respectively. 
For biological reasons we need the solutions nonnegative. Mathematical properties of the solutions lead us to study the system (5) in the closed set

$$
\Gamma=\left\{(S, I, V) \in \mathbf{R}_{+}^{3} \mid 0 \leq S+I \leq \frac{b_{1}}{\mu_{1}}, 0 \leq V \leq \frac{b_{2}}{\mu_{2}}, S \geq 0, I \geq 0\right\},
$$

where $\mathbf{R}_{+}^{\mathbf{3}}$ denotes the non-negative cone of $\mathbf{R}^{\mathbf{3}}$ including its lower dimensional faces. It can be verified that $\Gamma$ is positively invariant with respect to (5). We denote by $\partial \Gamma$ and $\Gamma^{0}$ the boundary and the interior of $\Gamma$ in $\mathbf{R}^{3}$, respectively.

\section{Equilibria and Bifurcation Analysis}

The disease-free equilibrium of system (5) is $E_{0}=\left(b_{1} /\right.$ $\left.\mu_{1}, 0,0\right)$. The endemic equilibria $E_{1}=\left(S^{*}, I^{*}, V^{*}\right)$ of system (5) can be deduced by the system,

$$
\begin{gathered}
b_{1}-\lambda_{1} S^{*} V^{*}\left[1+\alpha V^{*}\right]-\mu_{1} S^{*}=0, \\
\lambda_{1} S^{*} V^{*}\left[1+\alpha V^{*}\right]-\gamma I^{*}-\mu_{1} I^{*}=0, \\
\lambda_{2}\left[\frac{b_{2}}{\mu_{2}}-V^{*}\right] I^{*}-\mu_{2} V^{*}=0,
\end{gathered}
$$

which gives,

$$
S^{*}=\frac{b_{1}}{\lambda_{1} V^{*}\left(1+\alpha V^{*}\right)+\mu_{1}}, \quad I^{*}=\frac{\mu_{2} V^{*}}{\lambda_{2}\left(b_{2} / \mu_{2}-V^{*}\right)},
$$

and $V^{*}$ is the positive solution of the following equation,

$$
a_{1} V^{* 2}+a_{2} V^{*}+a_{3}=0
$$

where

$$
\begin{gathered}
a_{1}=\lambda_{1} \lambda_{2} b_{1} \alpha+\mu_{2} \lambda_{1} \alpha\left(\gamma+\mu_{1}\right), \\
a_{2}=\lambda_{1} \lambda_{2} b_{1}+\mu_{2} \lambda_{1}\left(\gamma+\mu_{1}\right)-\frac{\lambda_{1} \lambda_{2} b_{1} b_{2} \alpha}{\mu_{2}} \\
=\alpha \mu_{1} \mu_{2}\left(\gamma+\mu_{1}\right)\left(R_{1}^{*}-R_{0}\right), \\
a_{3}=\mu_{1} \mu_{2}\left(\gamma+\mu_{1}\right)-\frac{\lambda_{1} \lambda_{2} b_{1} b_{2}}{\mu_{2}}=\mu_{1} \mu_{2}\left(\gamma+\mu_{1}\right)\left(1-R_{0}\right), \\
R_{1}^{*}=\frac{\lambda_{1} \lambda_{2} b_{1}+\mu_{2} \lambda_{1}\left(\gamma+\mu_{1}\right)}{\alpha \mu_{1} \mu_{2}\left(\gamma+\mu_{1}\right)} \\
R_{0}=\frac{\lambda_{1} \lambda_{2} b_{1} b_{2}}{\mu_{1} \mu_{2}^{2}\left(\gamma+\mu_{1}\right)} .
\end{gathered}
$$

Thus, we observe that

$$
a_{1}>0 ; \quad a_{2}<0 \Longleftrightarrow R_{1}^{*}<R_{0} ; \quad a_{3}>0 \Longleftrightarrow R_{0}<1 .
$$

By the Descartes' rule of signs, it follows that there is a unique endemic equilibrium whenever $a_{3}<0$ and there are two endemic equilibria whenever $a_{3}>0, a_{2}<0$ and $a_{2}^{2}-4 a_{1} a_{3}>0$.
Furthermore, we observe that there is a bifurcation point when $a_{3}>0, a_{2}<0$ and $a_{2}^{2}-4 a_{1} a_{3}=0$. In fact the quantity $a_{2}^{2}-4 a_{1} a_{3}$ can be expressed in terms of new threshold $R_{0}^{*}$, where

$$
\begin{gathered}
R_{0}^{*}=\frac{M_{0}}{N_{0}} \\
M_{0}=\frac{2 \lambda_{1}^{2} \lambda_{2}^{2} b_{1}^{2} b_{2} \alpha}{\mu_{1} \mu_{2}\left(\gamma+\mu_{1}\right)}+\frac{2 \lambda_{1}^{2} \lambda_{2} b_{1} b_{2} \alpha}{\mu_{1}}+P_{0}, \\
N_{0}=\frac{\lambda_{1} \lambda_{2} b_{1} \mu_{2}^{2}+2 \lambda_{1} \mu_{2}^{3}\left(\gamma+\mu_{1}\right)}{b_{2}}+\frac{\lambda_{1} \mu_{2}^{4}\left(\gamma+\mu_{1}\right)^{2}}{\lambda_{2} b_{1} b_{2}} \\
+\lambda_{1} \lambda_{2} b_{1} b_{2} \alpha^{2}+P_{0}, \\
P_{0}=4 \lambda_{1} \lambda_{2} b_{1} \alpha \mu_{2}+4 \lambda_{1} \alpha \mu_{2}^{2}\left(\gamma+\mu_{1}\right) .
\end{gathered}
$$

By a little algebraic calculation, we have $a_{2}^{2}-4 a_{1} a_{3}>0$ whenever $R_{0}>R_{0}^{*}$. Taking into account the above consideration, we have the following theorem.

Theorem 1. System (7) admits a unique endemic equilibrium $E$ when $R_{0} \geq 1$; there are no endemic equilibria for $R_{0}<R_{0}^{*}$; there are two endemic equilibria, $E_{1}$ and $E_{2}$, for $R<R_{0}<1$ and $R=\max \left\{R_{0}^{*}, R_{1}^{*}\right\}$.

Next we analyze the stability of the disease-free equilibrium $E_{0}=\left(b_{1} / \mu_{1}, 0,0\right)$. The Jacobian matrix corresponding to $(7)$ is

$J(S, I, V)$

$$
=\left(\begin{array}{ccc}
-\lambda_{1} V(1+\alpha V)-\mu_{1} & 0 & -\lambda_{1} S(1+2 \alpha V) \\
\lambda_{1} V(1+\alpha V) & -\gamma-\mu_{1} & \lambda_{1} S(1+2 \alpha V) \\
0 & \lambda_{2}\left(\frac{b_{2}}{\mu_{2}}-V\right) & -\lambda_{2} I-\mu_{2}
\end{array}\right) .
$$

First, we observe that

$$
J\left(\frac{b_{1}}{\mu_{1}}, 0,0\right)=\left(\begin{array}{ccc}
-\mu_{1} & 0 & -\lambda_{1} \frac{b_{1}}{\mu_{1}} \\
0 & -\gamma-\mu_{1} & \lambda_{1} \frac{b_{1}}{\mu_{1}} \\
0 & \lambda_{2} \frac{b_{2}}{\mu_{2}} & -\mu_{2}
\end{array}\right),
$$

so that the eigenvalues $\xi$ are given by the roots of the following qubic equation

$$
\left(\mu_{1}+\xi\right)\left[\xi^{2}+\left(\gamma+\mu_{1}+\mu_{2}\right) \xi+\mu_{2}\left(\gamma+\mu_{1}\right)\left(1-R_{0}\right)\right]=0 .
$$

Hence if $R_{0}<1$, then the eigenvalues are all negatives and $E_{0}$ is locally asymptotically stable. If $R_{0}>1$, then two 
eigenvalues are negative and one is positive, so that $E_{0}$ is unstable.

Since there are no explicit expressions for equilibria $E_{1}$ and $E_{2}$ and the existence conditions for the two equilibria are very complex, it is very difficult to prove their stability by using the existing analytic methods.

Theorem 1 establishes that $R_{0}=1$ is a bifurcation value. In fact, across $R_{0}=1$ the disease free equilibrium changes its stability properties. Now, we investigate what kind of bifurcation occurs at $R_{0}=1$. In order to do that, we will make use of the result summarized below, which has been obtained in [6] and is based on the use of the center manifold theory [7]. ter $\phi$ :

Let us consider a general system of ODEs with a parame-

$$
\dot{x}=f(x, \phi) ; \quad f: R^{n} \times R \longrightarrow R^{n}, \quad f \in C^{2}\left(R^{n} \times R\right) .
$$

Without loss of generality, we assume that $x=0$ is an equilibrium for (16).

Theorem 2 (see (Castillo-Chavez and Song [6])). Assume:

(A1) $A=D_{x} f(0,0)$ is the linearization matrix of system (16) around the equilibrium $x=0$ with $\phi$ evaluated at 0 . Zero is a simple eigenvalue of $A$ and all other eigenvalues of $A$ have negative real parts.

(A2) Matrix A has a right eigenvector $\omega$ and a left eigenvector $\theta$ corresponding to the zero eigenvalue.

Let $f_{k}$ denotes the kth component of $f$ and,

$$
a=\sum_{k, i, j}^{n} \theta_{k} \omega_{i} \omega_{j} \frac{\partial^{2} f_{k}}{\partial x_{i} \partial x_{j}}(0,0), \quad b=\sum_{k, i, j}^{n} \theta_{k} \omega_{i} \frac{\partial^{2} f_{k}}{\partial x_{i} \partial \phi}(0,0) .
$$

Then the local dynamics of system (16) around $x=0$ are totally determined by $a$ and $b$

(i) $a>0, b>0$. When $\phi<0$, with $|\phi| \ll 1, x=$ 0 is locally asymptotically stable and there exists a positive unstable equilibrium; when $0<|\phi| \ll 1$, $x=0$ is unstable and there exists a negative and locally asymptotically stable equilibrium;

(ii) $a<0, b<0$. When $\phi<0$, with $|\phi| \ll 1, x=$ 0 is unstable; when $0<|\phi| \ll 1, x=0$ is locally asymptotically stable and there exists a positive unstable equilibrium;

(iii) $a>0, b<0$. When $\phi<0$, with $|\phi| \ll 1, x=0$ is unstable and there exists a locally asymptotically stable negative equilibrium; when $0<|\phi| \ll 1, x=0$ is stable and a positive unstable equilibrium appears;

(iv) $a<0, b\rangle 0$. When $\phi$ changes from negative to positive, $x=0$ changes its stability from stable to unstable. Correspondingly, a negative unstable equilibrium becomes positive and locally asymptotically stable.
It clearly appears that, at $\phi=0$ a transcritical bifurcation takes place: more precisely, when $a<0$ and $b>0$, such a bifurcation is forward; when $a>0$ and $b>0$ the bifurcation at $\phi=0$ is backward. We will apply Theorem 2 to show that system (7) may exhibit a backward bifurcation when $R_{0}=1$. We consider the disease-free equilibrium $E_{0}=\left(b_{1} / \mu_{1}, 0,0\right)$ and observe that the condition $R_{0}=1$ can be seen, in terms of the parameter $\lambda_{1}$, as $\lambda_{1}=\lambda^{*}=\mu_{1} \mu_{2}^{2}\left(\gamma+\mu_{1}\right) / \lambda_{2} b_{1} b_{2}$. The eigenvalues of the matrix

$$
J\left(E_{0}, \lambda^{*}\right)=\left(\begin{array}{ccc}
-\mu_{1} & 0 & -\lambda^{*} \frac{b_{1}}{\mu_{1}} \\
0 & -\gamma-\mu_{1} & \lambda^{*} \frac{b_{1}}{\mu_{1}} \\
0 & \lambda_{2} \frac{b_{2}}{\mu_{2}} & -\mu_{2}
\end{array}\right),
$$

are given by

$$
\xi_{1}=-\mu_{1}, \quad \xi_{2}=-\gamma-\mu_{1}-\mu_{2}, \quad \xi_{3}=0 .
$$

Thus $\xi_{3}=0$ is a simple zero eigenvalue of the matrix $J\left(E_{0}, \lambda^{*}\right)$ and the other eigenvalues are real and negative. Hence, when $\lambda_{1}=\lambda^{*}$ (or equivalently when $R_{0}=1$ ), the disease-free equilibrium $E_{0}$ is a nonhyperbolic equilibrium: the assumption (A1) of Theorem 2 is then verified.

Now we denote by $\omega=\left(\omega_{1}, \omega_{2}, \omega_{3}\right)^{T}$, a right eigenvector associated with the zero eigenvalue $\xi_{3}=0$. It follows:

$$
\begin{gathered}
-\mu_{1} \omega_{1}-\frac{\mu_{2}^{2}\left(\gamma+\mu_{1}\right)}{\lambda_{2} b_{2}} \omega_{3}=0, \\
-\left(\gamma+\mu_{1}\right) \omega_{2}+\frac{\mu_{2}^{2}\left(\gamma+\mu_{1}\right)}{\lambda_{2} b_{2}} \omega_{3}=0, \\
\frac{\lambda_{2} b_{2}}{\mu_{2}} \omega_{2}-\mu_{2} \omega_{3}=0,
\end{gathered}
$$

so that

$$
\omega=\left(-\frac{\gamma+\mu_{1}}{\mu_{1}}, 1, \frac{\lambda_{2} b_{2}}{\mu_{2}^{2}}\right)^{T} .
$$

Furthermore, the left eigenvector $\theta=\left(\theta_{1}, \theta_{2}, \theta_{3}\right)$ satisfying $\omega \cdot \theta=1$ is given by:

$$
\begin{gathered}
-\mu_{1} \theta_{1}=0, \\
-\left(\gamma+\mu_{1}\right) \theta_{2}+\frac{\lambda_{2} b_{2}}{\mu_{2}} \theta_{3}=0, \\
\frac{\mu_{2}^{2}\left(\gamma+\mu_{1}\right)}{\lambda_{2} b_{2}}\left(\theta_{2}-\theta_{1}\right)-\mu_{2} \theta_{3}=0, \\
\theta_{3}\left(\frac{\lambda_{2} b_{2}}{\mu_{2}\left(\gamma+\mu_{1}\right)}+\frac{\lambda_{2} b_{2}}{\mu_{2}^{2}}\right)=1 .
\end{gathered}
$$

Then, the left eigenvector $\theta$ turns out to be:

$$
\theta=\left(0, \frac{\mu_{2}}{\gamma+\mu_{1}+\mu_{2}}, \frac{\mu_{2}^{2}\left(\gamma+\mu_{1}\right)}{\lambda_{2} b_{2}\left(\gamma+\mu_{1}+\mu_{2}\right)}\right) .
$$


We can thus compute the coefficient $a$ and $b$ defined in Theorem 2, that is,

$$
\begin{aligned}
& a=\sum_{k, i, j=1}^{3} \theta_{k} \omega_{i} \omega_{j} \frac{\partial^{2} f_{k}}{\partial x_{i} \partial x_{j}}\left(E_{0}, \lambda^{*}\right), \\
& b=\sum_{k, i=1}^{3} \theta_{k} \omega_{i} \frac{\partial^{2} f_{k}}{\partial x_{i} \partial \lambda_{1}}\left(E_{0}, \lambda^{*}\right) .
\end{aligned}
$$

Taking into account of system (5) and considering in $a$ and $b$ only the nonzero derivatives for the terms $\left(\partial^{2} f_{k} /\right.$ $\left.\partial x_{i} \partial x_{j}\right)\left(E_{0}, \lambda^{*}\right)$ and $\left(\partial^{2} f_{k} / \partial x_{i} \partial \lambda_{1}\right)\left(E_{0}, \lambda^{*}\right)$, it follows that:

$$
\begin{aligned}
a=2 & \theta_{1} \omega_{1} \omega_{3} \frac{\partial^{2} f_{1}}{\partial S \partial V}\left(E_{0}, \lambda^{*}\right)+\theta_{1} \omega_{3}^{2} \frac{\partial^{2} f_{1}}{\partial V^{2}}\left(E_{0}, \lambda^{*}\right) \\
& +2 \theta_{2} \omega_{1} \omega_{3} \frac{\partial^{2} f_{2}}{\partial S \partial V}\left(E_{0}, \lambda^{*}\right)+\theta_{2} \omega_{3}^{2} \frac{\partial^{2} f_{2}}{\partial V^{2}}\left(E_{0}, \lambda^{*}\right) \\
& +2 \theta_{3} \omega_{2} \omega_{3} \frac{\partial^{2} f_{3}}{\partial V \partial I}\left(E_{0}, \lambda^{*}\right), \\
b= & \theta_{1} \omega_{1} \frac{\partial^{2} f_{1}}{\partial S \partial \lambda_{1}}\left(E_{0}, \lambda^{*}\right)+\theta_{1} \omega_{3} \frac{\partial^{2} f_{1}}{\partial V \partial \lambda_{1}}\left(E_{0}, \lambda^{*}\right) \\
& +\theta_{2} \omega_{1} \frac{\partial^{2} f_{2}}{\partial S \partial \lambda_{1}}\left(E_{0}, \lambda^{*}\right)+\theta_{2} \omega_{3} \frac{\partial^{2} f_{2}}{\partial V \partial \lambda_{1}}\left(E_{0}, \lambda^{*}\right) .
\end{aligned}
$$

In view of (21) and (23), we get

$$
\begin{gathered}
a=\frac{2 \lambda_{2}}{\mu_{1} \mu_{2}^{3}\left(\gamma+\mu_{1}+\mu_{2}\right)}\left[\lambda_{1} \lambda_{2} b_{1} b_{2}^{2} \alpha\right. \\
\left.-\mu_{2}^{2}\left(\gamma+\mu_{1}\right)\left(\lambda_{1} b_{2}+\mu_{1} \mu_{2}\right)\right], \\
b=\frac{\lambda_{2} b_{1} b_{2}}{\mu_{1} \mu_{2}\left(\gamma+\mu_{1}+\mu_{2}\right)} .
\end{gathered}
$$

Observe that the coefficient $b$ is always positive so that, according to Theorem 2 , it is the sign of the coefficient $a$ which decides the local dynamics around the disease-free equilibrium for $\lambda_{1}=\lambda^{*}$.

Let us introduce

$$
\delta=\frac{\lambda_{1} \lambda_{2} b_{1} b_{2}^{2} \alpha}{\mu_{2}^{2}\left(\gamma+\mu_{1}\right)\left(\lambda_{1} b_{2}+\mu_{1} \mu_{2}\right)} .
$$

The coefficient $a$ is positive if and only if $\delta>1$. In this case, the direction of the bifurcation of system (5) at $R_{0}=1$ is backward. So we summarize the above results in the following theorem.

Theorem 3. If $\delta>1$, system (5) exhibits a backward bifurcation when $R_{0}=1$. If $\delta<1$, system (5) exhibits a forward bifurcation when $R_{0}=1$.

The local dynamics in the neighborhood of the bifurcation value $R_{0}=1$ is described in the former case by the (i) of Theorem 2 and in the later case by the (iv) of the same theorem.

\section{Global Stability Analysis}

In Section 3 it has been shown that $R_{0} \geq 1$ implies the existence and uniqueness of the endemic equilibrium $E$. The stability analysis of $E$ will be here performed through the geometric approach to global stability due to Li and Muldowney [8]. The method has been summarized in the Appendix.

System (5), under the assumption $R_{0}>1$, satisfies conditions ( $\mathrm{H} 1)$ and $(\mathrm{H} 2)$ in Appendix. In fact, when $R_{0}>$ 1 , then $E_{0}$ is unstable. The instability of $E_{0}$, together with $E_{0} \in \partial \Gamma$, implies the uniform persistence, that is, there exists a constant $c>0$ such that:

$$
\begin{gathered}
\lim _{t \rightarrow+\infty} \inf S(t)>c, \quad \lim _{t \rightarrow+\infty} \inf I(t)>c, \\
\lim _{t \rightarrow+\infty} \inf V(t)>c .
\end{gathered}
$$

The uniform persistence is equivalent to the existence of a compact set in the interior of $\Gamma$ which is absorbing for (5). Thus, (H1) is verified. Moreover, as previously shown, $E$ is the only equilibrium in the interior of $\Gamma$, so that $(\mathrm{H} 2)$ is verified, too. Now it remains to find conditions for which the Bendixson criterion given by Theorem A.4 is verified. Taking into account of the Jacobian matrix (13), we obtain the second additive compound matrix $J^{[2]}(S, I, V)$,

$$
J^{[2]}=\left(\begin{array}{ccc}
J_{11}^{[2]} & \lambda_{1} S(1+2 \alpha V) & \lambda_{1} S(1+2 \alpha V) \\
\lambda_{2}\left(\frac{b_{2}}{\mu_{2}}-V\right) & J_{22}^{[2]} & 0 \\
0 & \lambda_{1} V(1+\alpha V) & J_{33}^{[2]}
\end{array}\right) \text {, }
$$

where

$$
\begin{gathered}
J_{11}^{[2]}=-\lambda_{1} V(1+\alpha V)-\gamma-2 \mu_{1}, \\
J_{22}^{[2]}=-\lambda_{1} V(1+\alpha V)-\mu_{1}-\lambda_{2} I-\mu_{2}, \\
J_{33}^{[2]}=-\gamma-\mu_{1}-\lambda_{2} I-\mu_{2} .
\end{gathered}
$$

We consider the following function

$$
P=P(S, I, V)=\operatorname{diag}\left\{\frac{I}{V}, \frac{I}{V}, \frac{I}{V}\right\} .
$$

So that

$$
\begin{gathered}
P_{f}=\operatorname{diag}\left\{\frac{I^{\prime} V-V^{\prime} I}{V^{2}}, \frac{I^{\prime} V-V^{\prime} I}{V^{2}}, \frac{I^{\prime} V-V^{\prime} I}{V^{2}}\right\}, \\
P_{f} P^{-1}=\operatorname{diag}\left\{\frac{I^{\prime}}{I}-\frac{V^{\prime}}{V}, \frac{I^{\prime}}{I}-\frac{V^{\prime}}{V}, \frac{I^{\prime}}{I}-\frac{V^{\prime}}{V}\right\} .
\end{gathered}
$$

Therefore

$$
B=P_{f} P^{-1}+P J^{[2]} P^{-1}=\left(\begin{array}{ll}
B_{11} & B_{12} \\
B_{21} & B_{22}
\end{array}\right),
$$


where

$$
\begin{gathered}
B_{11}=\frac{I^{\prime}}{I}-\frac{V^{\prime}}{V}-\lambda_{1} V(1+\alpha V)-2 \mu_{1}-\gamma, \\
B_{12}=\left[\lambda_{1} S(1+2 \alpha V), \lambda_{1} S(1+2 \alpha V)\right], \\
B_{21}=\left[\lambda_{2}\left(\frac{b_{2}}{\mu_{2}}-V\right), 0\right]^{T}, \\
B_{22} \\
=\left[\begin{array}{cc}
\frac{I^{\prime}}{I}-\frac{V^{\prime}}{V}-\lambda_{1} V(1+\alpha V)-\mu_{1}-\lambda_{2} I-\mu_{2} & 0 \\
\lambda_{1} V(1+\alpha V) & \frac{I^{\prime}}{I}-\frac{V^{\prime}}{V}-\gamma-\mu_{1}-\lambda_{2} I-\mu_{2}
\end{array}\right] .
\end{gathered}
$$

Consider now the norm in $R^{3}$ as

$$
\left|u_{1}, u_{2}, u_{3}\right|=\max \left\{\left|u_{1}\right|,\left|u_{2}\right|+\left|u_{3}\right|\right\},
$$

where $\left(u_{1}, u_{2}, u_{3}\right)$ denotes the vector in $R^{3}$ and denote by $\mu$ the Lozinskii measure with respect to this norm. It follows

$$
\mu(B) \leq \sup \left\{g_{1}, g_{2}\right\}=\sup \left\{\mu_{1}\left(B_{11}\right)+\left|B_{12}\right|, \mu_{1}\left(B_{22}\right)+\left|B_{21}\right|\right\} \text {, }
$$

where $\left|B_{12}\right|,\left|B_{21}\right|$ are matrix norms with respect to the $l_{1}$ vector norm, and $\mu_{1}$ denotes the Lozinskii measure with respect to the $l_{1}$ norm. More specifically,

$$
\begin{gathered}
\mu_{1}\left(B_{11}\right)=\frac{I^{\prime}}{I}-\frac{V^{\prime}}{V}-\lambda_{1} V(1+\alpha V)-\gamma-2 \mu_{1}, \\
\left|B_{12}\right|=\lambda_{1} S(1+2 \alpha V), \\
\left|B_{21}\right|=\lambda_{2}\left(\frac{b_{2}}{\mu_{2}}-V\right), \\
\mu_{1}\left(B_{22}\right)=\frac{I^{\prime}}{I}-\frac{V^{\prime}}{V}-\mu_{1}-\lambda_{2} I-\mu_{2} .
\end{gathered}
$$

Therefore,

$$
\begin{gathered}
g_{1}=\frac{I^{\prime}}{I}-\frac{V^{\prime}}{V}-\lambda_{1} V(1+\alpha V)-2 \mu_{1}-\gamma+\lambda_{1} S(1+2 \alpha V), \\
g_{2}=\frac{I^{\prime}}{I}-\frac{V^{\prime}}{V}-\mu_{1}-\lambda_{2} I-\mu_{2}+\lambda_{2}\left(\frac{b_{2}}{\mu_{2}}-V\right) .
\end{gathered}
$$

Observe that system (5) provides the following equalities

$$
\frac{V^{\prime}}{V}=\frac{\lambda_{2} b_{2} I}{\mu_{2} V}-\lambda_{2} I-\mu_{2}
$$

Substituting (40) into (38) and (40) into (39), respectively, we have

$$
\begin{aligned}
g_{1}= & \frac{I^{\prime}}{I}-\frac{\lambda_{2} b_{2} I}{\mu_{2} V}+\lambda_{2} I+\mu_{2}-\lambda_{1} V(1+\alpha V) \\
& -2 \mu_{1}-\gamma+\lambda_{1} S(1+2 \alpha V) \\
\leq & \frac{I^{\prime}}{I}+\lambda_{2} I+\mu_{2}-\lambda_{1} V(1+\alpha V)-2 \mu_{1}-\gamma+\lambda_{1} S(1+2 \alpha V), \\
g_{2}= & \frac{I^{\prime}}{I}-\frac{\lambda_{2} b_{2} I}{\mu_{2} V}-\mu_{1}+\lambda_{2}\left(\frac{b_{2}}{\mu_{2}}-V\right) \\
\leq & \frac{I^{\prime}}{I}-\mu_{1}+\lambda_{2}\left(\frac{b_{2}}{\mu_{2}}-V\right) .
\end{aligned}
$$

Thus, (36) implies

$$
\mu(B) \leq \frac{I^{\prime}}{I}-\mu_{1}+\Lambda,
$$

where

$$
\begin{aligned}
\Lambda=\max \{ & \lambda_{2} I+\mu_{2}-\lambda_{1} V(1+\alpha V)-\mu_{1}-\gamma \\
& \left.+\lambda_{1} S(1+2 \alpha V), \lambda_{2}\left(\frac{b_{2}}{\mu_{2}}-V\right)\right\} .
\end{aligned}
$$

In view of $c \leq S, I \leq b_{1} / \mu_{1}$ and $c \leq V \leq b_{2} / \mu_{2}$, we can deduce that if

$$
\begin{aligned}
\lambda_{2}\left(\frac{b_{2}}{\mu_{2}}-c\right) & <\mu_{1}, \\
\lambda_{2} \frac{b_{1}}{\mu_{1}}+\mu_{2}+\lambda_{1} \frac{b_{1}}{\mu_{1}}\left(1+2 \alpha \frac{b_{2}}{\mu_{2}}\right) & <\lambda_{1} c(1+\alpha c)+\mu_{1}+\gamma,
\end{aligned}
$$

then

$$
\mu(B) \leq \frac{I^{\prime}}{I}+\Pi
$$

where

$$
\begin{aligned}
\Pi=\max \{ & \lambda_{2} \frac{b_{1}}{\mu_{1}}+\mu_{2}+\lambda_{1} \frac{b_{1}}{\mu_{1}}\left(1+2 \alpha \frac{b_{2}}{\mu_{2}}\right)-\lambda_{1} c(1+\alpha c) \\
& \left.-\mu_{1}-\gamma, \lambda_{2}\left(\frac{b_{2}}{\mu_{2}}-c\right)-\mu_{1}\right\}<0 .
\end{aligned}
$$

Hence

$$
\frac{1}{t} \int_{0}^{t} \mu(B) d s \leq \frac{1}{t} \log \frac{I(t)}{I(0)}+\Pi,
$$

and the Bendixson criterion given by Theorem A.4 is thus verified. The discussions above may be summarized as follows.

Theorem 4. If $R_{0} \geq 1$, then system (5) admits a unique endemic equilibrium $E$. It is globally asymptotically stable with respect to solutions of (5) initiating in the interior of $\Gamma$, provided that inequality (44) holds true. 


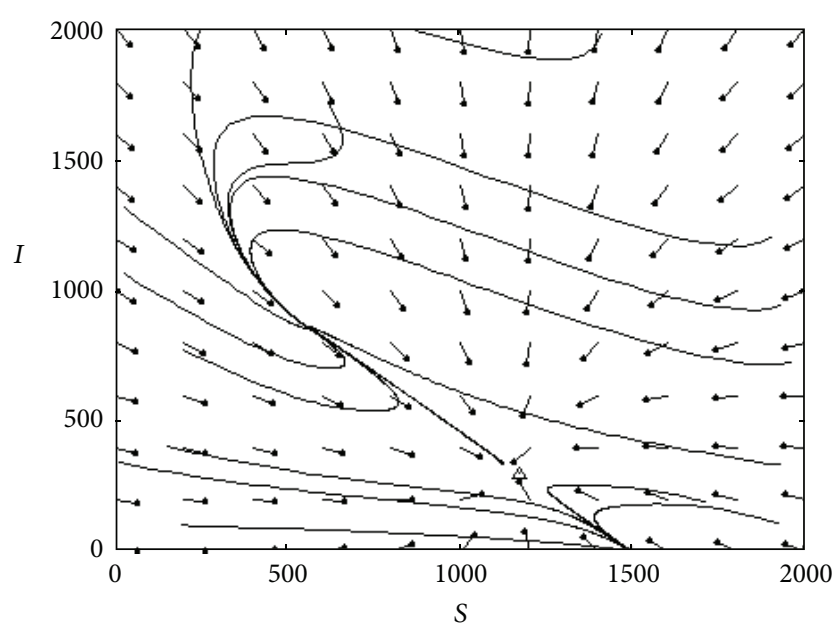

FIGURE 1: Phase plot of $I$ verses $S$ showing bistability when $R<R_{0}<$ 1 and $\delta>1$ for the parameter values $b_{1}=240, \lambda_{1}=0.00012, \alpha=$ $0.08, \mu_{1}=0.16, \gamma=0.015, \lambda_{2}=0.0001, b_{2}=550, \mu_{2}=0.5$.

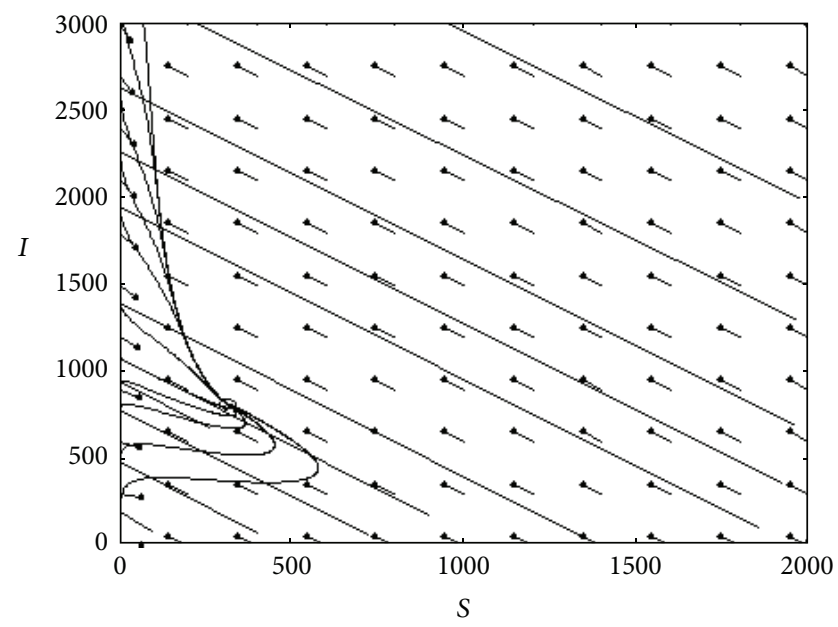

Figure 2: Phase plot of $I$ verses $S$ showing stability of unique endemic equilibrium point when $R_{0}>1$ and $\delta>1$ for the parameter values $b_{1}=80, \lambda_{1}=0.0004, \alpha=0.04, \mu_{1}=0.06, \gamma=0.015, \lambda_{2}=$ $0.0002, b_{2}=200, \mu_{2}=0.5$.

\section{Simulation}

The system (5) is simulated for various sets of parameters using the package XPP [9]. In Figures 1-4, $(S, I)$ phase planes are drawn which confer the existence and the stability of different equilibria of the system (5). Here Figure 1 corresponds to the situation stated in Theorem 1 , where $R<R_{0}<$ 1 and we get two endemic equilibria $E_{1}$ and $E_{2}$ and one disease free equilibria $E_{0}(1500,0,0)$. And for $\delta>1$, it is found that disease free equilibrium $E_{0}(1500,0,0)$ and the endemic equilibrium point with largest infective population $E_{2}(557.678,861.551,161.682)$ are stable and the equilibrium point $E_{1}(1170.057,301.662,62.589)$ is unstable.

Figure 2 corresponds to the situation when $R_{0}>1$ and also $\delta>1$ and in this case we get unique stable endemic equilibrium point and unstable disease free equilibrium point

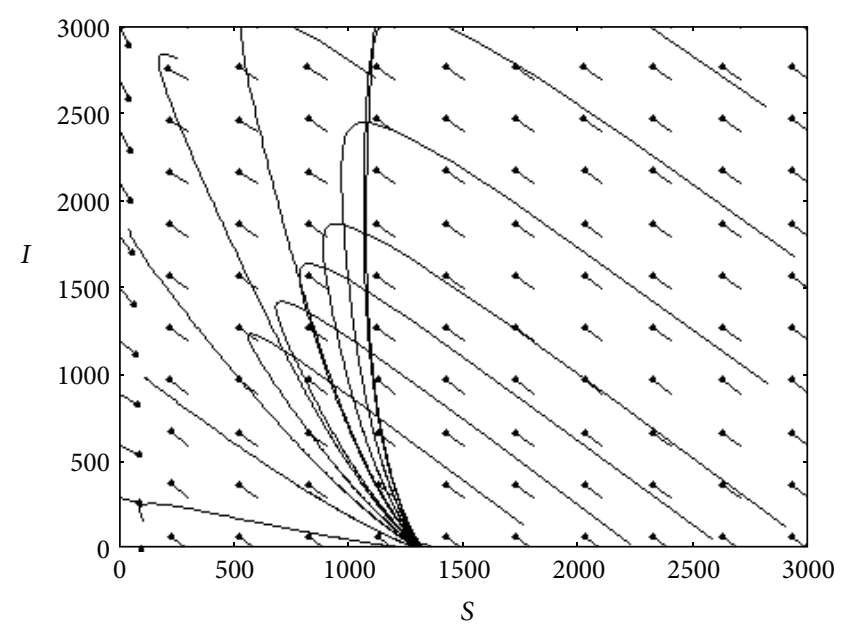

FIgURE 3: Phase plot of $I$ verses $S$ showing stability of unique feasible disease free equilibrium point when $R_{0}<R_{0}^{*}<1$ for the parameter values $b_{1}=80, \lambda_{1}=0.00001, \alpha=0.04, \mu_{1}=0.06, \gamma=0.015, \lambda_{2}=$ $0.0002, b_{2}=200, \mu_{2}=0.5$.

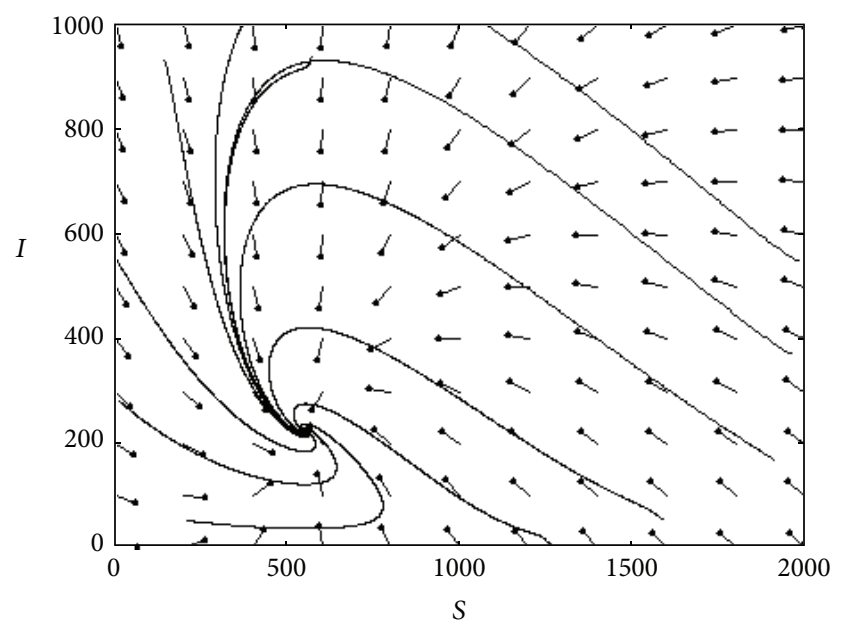

FIGURE 4: Phase plot of $I$ verses $S$ showing stability of unique feasible endemic equilibrium point and instablity of disease free equilibrium point when $\delta<1, R_{0}>1$ for the parameter values $b_{1}=10, \lambda_{1}=$ 0.0005, $\alpha=0.01, \mu_{1}=0.01, \gamma=0.01, \lambda_{2}=0.0003, b_{2}=60, \mu_{2}=$ 0.5 .

$E_{0}$. Figure 3 shows the stability of unique feasible disease free equilibrium $E_{0}(1333.3,0,0)$ for $R_{0}<R_{0}^{*}<1$. For $\delta<1$, and $R_{0} \geq 1$ we get two feasible equilibria: the unstable disease free equilibria and a unique endemic equilibrium which is shown in Figure 4. This fact is more clear from the bifurcation Figure 5, which is obtained by considering $\lambda_{1}$ as the critical parameter. The horizontal axis is labelled with the appropriate value of the reproduction number $R_{0}$ corresponding to this bifurcation parameter $\lambda_{1}$. For $0<R_{0}<1$ we get stable infection free equilibrium point $E_{0}$ and at $R_{0}=1$ we get forward bifurcation, that is, for $R_{0}>1$ we get unstable disease free equilibrium and unique stable endemic equilibrium point. Figure 6 is showing the backward bifurcation for the parameter values satisfying $\delta>1$. This diagram too is 


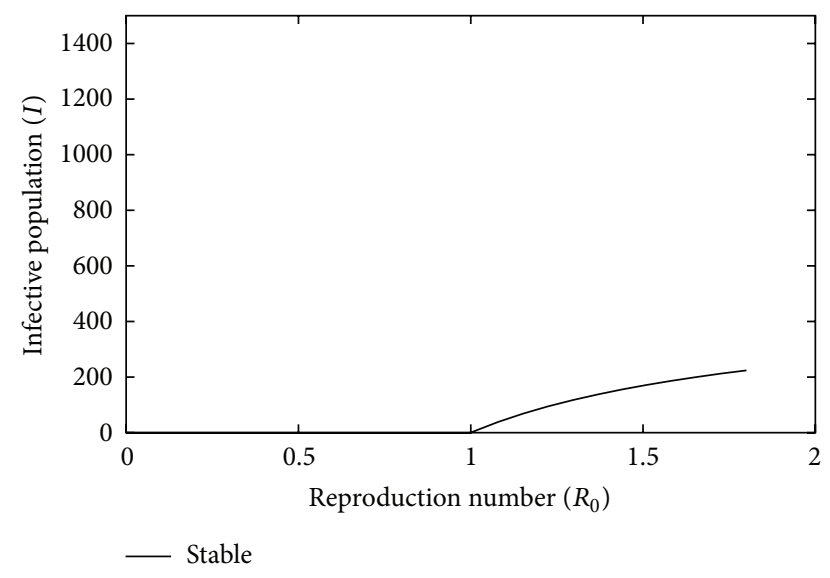

FIGURE 5: Forward bifurcation diagram for $\delta<1$ for the parameter values $b_{1}=10, \lambda_{1}=0.0005, \alpha=0.01, \mu_{1}=0.01, \gamma=0.01, \lambda_{2}=$ $0.0003, b_{2}=60, \mu_{2}=0.5$.

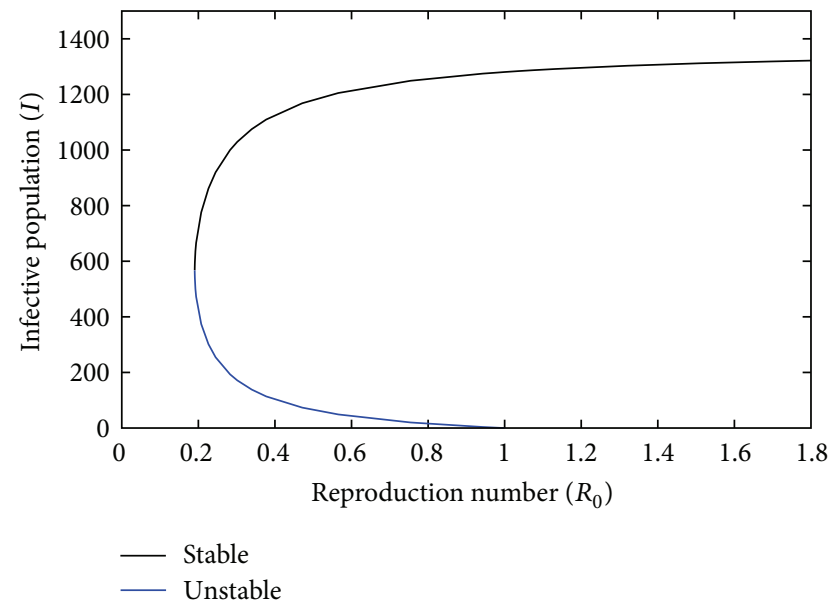

FIGURE 6: Backward bifurcation diagram for $\delta>1$ for the parameter values $b_{1}=240, \lambda_{1}=0.00012, \alpha=0.08, \mu_{1}=0.16, \gamma=0.015, \lambda_{2}=$ $0.0001, b_{2}=550, \mu_{2}=0.5$.

obtained by considering $\lambda_{1}$ as the critical parameter and horizontal axis is labelled with the appropriate value of the reproduction number $R_{0}$ corresponding to this bifurcation parameter $\lambda_{1}$. It is observed that when the reproduction number $R_{0}$ is between 0 to 0.190526 , the infection free equilibrium alone is stable, for $0.1905261<R_{0}<1$ we have bistability where either the infection free equilibrium is stable or the equilibrium $E_{2}$ is stable. Again by increasing $\lambda_{1}$ we get $R_{0}>1$ and we have unique stable endemic equilibrium point and unstable disease free equilibrium $E_{0}$. The equilibrium $E_{1}$ when it exists is always saddle. As backward bifurcation is quite significant in this figure, so it seems that it can arise for biologically reasonable parameter values too.

\section{Conclusions}

In this paper, a dengue disease epidemic model with nonlinear incidence has been analyzed. If $R_{0}<1$, the diseasefree equilibrium $E_{0}$ is locally asymptotically stable, that is, the disease dies out. We find the condition under which the system exhibits backward bifurcation at $R_{0}=1$. The stability of two endemic equilibria in the case of backward bifurcation for $R_{0}<1$ is not discussed analytically but we have shown these by numerical simulations. It is found that the endemic equilibrium with the largest infective population is stable, while the endemic equilibrium with less infective population is unstable. In the case of a unique endemic equilibrium, the global stability analysis has been performed. A generalization of the Poincaré-Bendixson criterion has been used. The sufficient conditions that we found are not completely satisfactory, as they involve the constant of uniform persistence $c$. How to implement the geometric approach to give better stability conditions for epidemic models with convex incidence rate appears to be an open and stimulating challenge.

\section{Appendix}

Let $x \mapsto f(x) \in R^{n}$ be a $C^{1}$ function for $x$ in an open set $D \subset R^{n}$. Consider the differential equation

$$
x^{\prime}=f(x) .
$$

Denote by $x\left(t, x_{0}\right)$ the solution of (A.1) such that $x\left(0, x_{0}\right)=$ $x_{0}$. We take the following two assumptions.

(H1) There exits a compact absorbing set $K \subset D$.

(H2) Equation (A.1) has a unique equilibrium $\bar{x}$ in $D$.

The equilibrium $\bar{x}$ is said to be globally stable in $D$ if it is locally stable and all trajectories in $D$ converge to $\bar{x}$. The following global-stability problem is formulated in [8].

Theorem A.1. Under the assumptions (H1) and (H2), the global stability of $\bar{x}$ with respect to $D$ is implied by its local stability.

Assumptions (H1) and (H2) are satisfied if $\bar{x}$ is global stability in $D$. For $n \geq 2$, a Bendixson criterion is a condition satisfied by $f$ which precludes the existence of nonconstant periodic solution of (A.1). A Bendixson criterion is said to be robust under $C^{1}$ local perturbations of $f$ at $x_{1} \in D$ if, for sufficiently small $\epsilon \geq 0$ and neighborhood $U$ of $x_{1}$, it is also satisfied by $g \in C^{1}\left(D \rightarrow R^{n}\right)$ such that the support $\operatorname{supp}(f-g) \subset U$ and $|f-g|_{C^{1}}<\epsilon$, where

$$
|f-g|_{C^{1}}=\sup \left\{|f(x)-g(x)|+\left|\frac{\partial f}{\partial x}(x)-\frac{\partial g}{\partial x}(x)\right|: x \in D\right\} .
$$

Such $g$ will be called local $\epsilon$-perturbations of $f$ at $x_{1}$. It is easy to see that the classic Bendixson's condition div $f(x)<0$ for $n=2$ is robust under $C^{1}$ local perturbations of at each $x_{1} \in$ $R^{2}$. Bendixson's condition for higher dimensional system that are $C^{1}$ robust are discussed in $[8,10,11]$.

A point $x_{0} \in D$ is wandering for (A.1) if there exists a neighborhood $U$ of $x_{0}$ and $T>0$ such that $U \cap x(t, U)$ is empty for all $t>T$. Thus, for example, all equilibria and limit points are nonwandering. The following is a version of the local $C^{1}$ Closing lemma of Pugh (see $\left.[12,13]\right)$. 
Lemma A.2. Let $f \in C^{1}\left(D \rightarrow R^{n}\right)$. Assume that all positive semitrajectories of (A.1) are bounded. Suppose that $x_{0}$ is a nonwandering point of (A.1) and that $f\left(x_{0}\right) \neq 0$. Then, for each neighborhood $U$ of $x_{0}$ and $\epsilon>0$, there exists a $C^{1}$ local- $\epsilon$ perturbation $g$ of $f$ at $x_{0}$ such that

(a) $\operatorname{supp}(f-g) \subset U$ and

(b) the perturbed system $x^{\prime}=g(x)$ has a nonconstant periodic solution whose trajectory passes through $x_{0}$.

The following general global-stability principle is established in [8].

Theorem A.3. Suppose that assumptions (H1) and (H2) hold. Assume that (A.1) satisfies a Bendixson criterion that is robust under $C^{1}$ local perturbations of $f$ at all nonequilibrium nonwandering points for (A.1). Then $\bar{x}$ is globally stable in $D$ provided it is stable.

The main idea of the proof in [8] for Theorem A.3 is as follows: suppose that system (A.1) satisfies a Bendixson criterion. Then it does not have any nonconstant periodic solutions. Moreover, the robustness of the Bendixson criterion implies that all nearby differential equations have nonconstant periodic solutions. Thus by Lemma A.2, all of nonwandering points of (A.1) in $D$ must be equilibria. In particular, each omega limit point in $D$ must be an equilibrium. Therefore $\omega\left(x_{0}\right)=\{\bar{x}\}$ for all $x_{0} \in D$ since $\bar{x}$ is the only equilibrium in $D$.

A method of deriving a Bendixson criterion in $R^{n}$ is developed in [14]. The idea is to show that second compound equation

$$
z^{\prime}(t)=\frac{\partial f^{[2]}}{\partial x}\left(x\left(t, x_{0}\right)\right) z(t)
$$

with respect to a solution $x\left(t, x_{0}\right) \subset D$ to (A.1), is uniformly asymptotically stable, and the exponential decay rate of all solutions to (A.3) is uniform for $x_{0}$ in each compact subset of $D$. Here $\partial f^{[2]} / \partial x$ is second additive compound matrix of the Jacobian matrix $\partial f / \partial x$. Generally speaking, for an $n \times n$ matrix $J=\left(J_{i j}\right), J^{[2]}$ is a $\left(\begin{array}{c}n \\ 2\end{array}\right) \times\left(\begin{array}{c}n \\ 2\end{array}\right)$ matrix and in the special case $n=3$, one has

$$
J^{[2]}=\left(\begin{array}{ccc}
J_{11}+J_{22} & a_{23} & -J_{13} \\
J_{32} & J_{11}+J_{33} & J_{12} \\
-J_{31} & J_{21} & J_{22}+J_{33}
\end{array}\right) .
$$

$\partial f^{[2]} / \partial x$ is a $\left(\begin{array}{l}n \\ 2\end{array}\right) \times\left(\begin{array}{c}n \\ 2\end{array}\right)$ matrix, and thus (A.3) is a linear system of dimension $\left(\begin{array}{l}n \\ 2\end{array}\right)$. If $D$ is simply connected, the above mentioned stability property of (A.3) implies the exponential decay of the surface area of any compact $2 d$ surface in $D$, which in turn precludes the existence of any invariant simply closed rectifiable curve in $D$, including periodic orbits. The required uniform asymptotic stability of system (A.3) can be proved by constructing a suitable Lyapunov function.
Let $x \mapsto P(x)\left(\begin{array}{c}n \\ 2\end{array}\right) \times\left(\begin{array}{c}n \\ 2\end{array}\right)$ matrix-valued function that is $C^{1}$ for $x \in D$. Assume that $P^{-1}(x)$ exists and is continuous for $x \in K$, the compact absorbing set. A quantity $\bar{q}_{2}$ is defined as

$$
\bar{q}_{2}=\limsup _{t \rightarrow \infty} \sup _{x_{0} \in K} \frac{1}{t} \int_{0}^{t} \mu\left(B\left(x\left(s, x_{0}\right)\right)\right) d s,
$$

where

$$
B=P_{f} P^{-1}+P \frac{\partial f^{[2]}}{\partial x} P^{-1},
$$

and the matrix $P_{f}$ is obtained by replacing each entry $p_{i j}$ of $P$ by its derivative in the direction of $f, p_{i j f}$. The quantity $\mu(B)$ is the Lozinskii measure of $B$ with respect to a vector norm $|\cdot|$ in $R^{N}, N=\left(\begin{array}{c}n \\ 2\end{array}\right)$, defined by

$$
\mu(B)=\lim _{h \rightarrow 0^{+}} \frac{|I+h B|-1}{h},
$$

see [14, p. 41]. It is shown in [8] that, if $D$ is simply connected, the condition $\bar{q}_{2}<0$ rules out the presence of any orbit that gives rise to a simple closed rectifiable curve that is invariant for (A.1), such as periodic orbits, homoclinic orbits, and heteroclinic cycles. Moreover, it is robust under $C^{1}$ local perturbations of $f$ near any nonequilibrium point that is nonwandering. In particular, the following global stability result is proved in Theorem 3.5 of [8].

Theorem A.4. Assume that $D$ is simply connected and that assumptions (H1), (H2) hold. Then the unique equilibrium $\bar{x}$ of (A.1) is globally stable in D if $\bar{q}_{2}<0$.

\section{Acknowledgments}

The authors are very grateful to two referees for their valuable comments and suggestions which led to an improvement of their original paper. They also thank the Handling Editor and all the editorial staff for the help and support provided to them. This work is supported by the NSF of China (no. 11271314) and NSF of Henan Province (no. 112300410058).

\section{References}

[1] Y. Xiao and S. Tang, "Dynamics of infection with nonlinear incidence in a simple vaccination model," Nonlinear Analysis. Real World Applications, vol. 11, no. 5, pp. 4154-4163, 2010.

[2] L.-M. Cai and X.-Z. Li, "Global analysis of a vector-host epidemic model with nonlinear incidences," Applied Mathematics and Computation, vol. 217, no. 7, pp. 3531-3541, 2010.

[3] Z. Hu, W. Ma, and S. Ruan, "Analysis of SIR epidemic models with nonlinear incidence rate and treatment," Mathematical Biosciences, vol. 238, no. 1, pp. 12-20, 2012.

[4] L. Li, G.-Q. Sun, and Z. Jin, "Bifurcation and chaos in an epidemic model with nonlinear incidence rates," Applied Mathematics and Computation, vol. 216, no. 4, pp. 1226-1234, 2010.

[5] M. Ozair, A. A. Lashari, I. H. Jung, and K. O. Okosun, "Stability analysis and optimal control of a vector-borne disease with nonlinear incidence," Discrete Dynamics in Nature and Society, vol. 2012, Article ID 595487, 21 pages, 2012. 
[6] C. Castillo-Chavez and B. Song, "Dynamical models of tuberculosis and their applications," Mathematical Biosciences and Engineering, vol. 1, no. 2, pp. 361-404, 2004.

[7] J. Guckenheimer and P. Holmes, Nonlinear Oscillations, Dynamical Systems, and Bifurcations of Vector Fields, vol. 42 of Applied Mathematical Sciences, Springer, New York, NY, USA, 1983.

[8] M. Y. Li and J. S. Muldowney, "A geometric approach to globalstability problems," SIAM Journal on Mathematical Analysis, vol. 27, no. 4, pp. 1070-1083, 1996.

[9] B. Ermentrout, Simulating, Analyzing, and Animating Dynamical Systems: A Guide to XPPAUT for Researchers and Students, vol. 14 of Software, Environments, and Tools, SIAM, Philadelphia, Pa, USA, 2002.

[10] M. Y. Li and J. S. Muldowney, “On R. A. Smith's autonomous convergence theorem," The Rocky Mountain Journal of Mathematics, vol. 25, no. 1, pp. 365-379, 1995.

[11] Y. Li and J. S. Muldowney, “On Bendixson's criterion," Journal of Differential Equations, vol. 106, no. 1, pp. 27-39, 1993.

[12] C. C. Pugh, "An improved closing lemma and a general density theorem," American Journal of Mathematics, vol. 89, pp. 10101021, 1967.

[13] C. C. Pugh and C. Robinson, "The $C^{1}$ closing lemma, including Hamiltonians," Ergodic Theory and Dynamical Systems, vol. 3, no. 2, pp. 261-313, 1983.

[14] W. A. Coppel, Stability and Asymptotic Behavior of Differential Equations, D. C. Heath and Co., Boston, Mass, USA, 1965. 


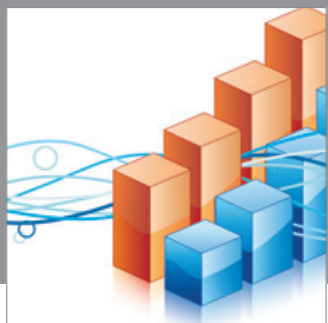

Advances in

Operations Research

mansans

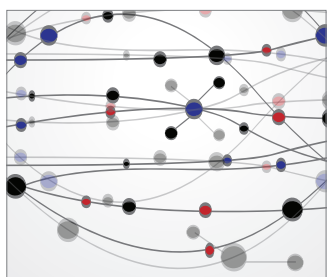

The Scientific World Journal
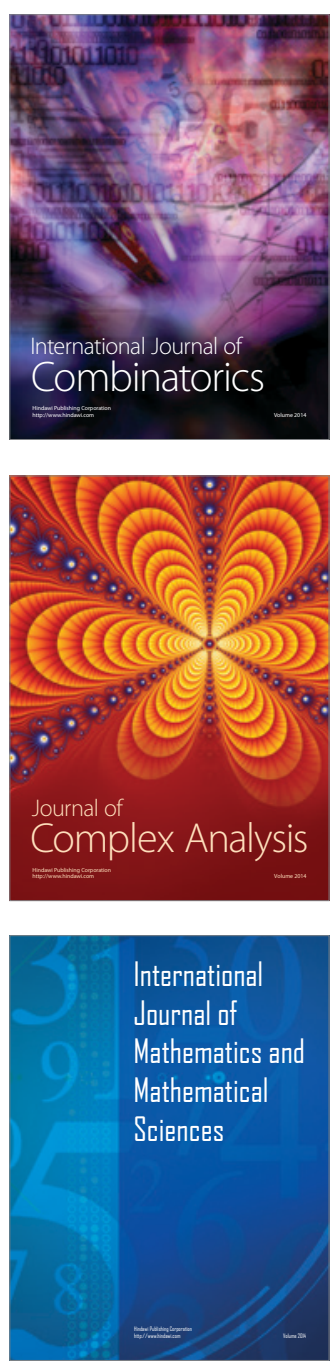
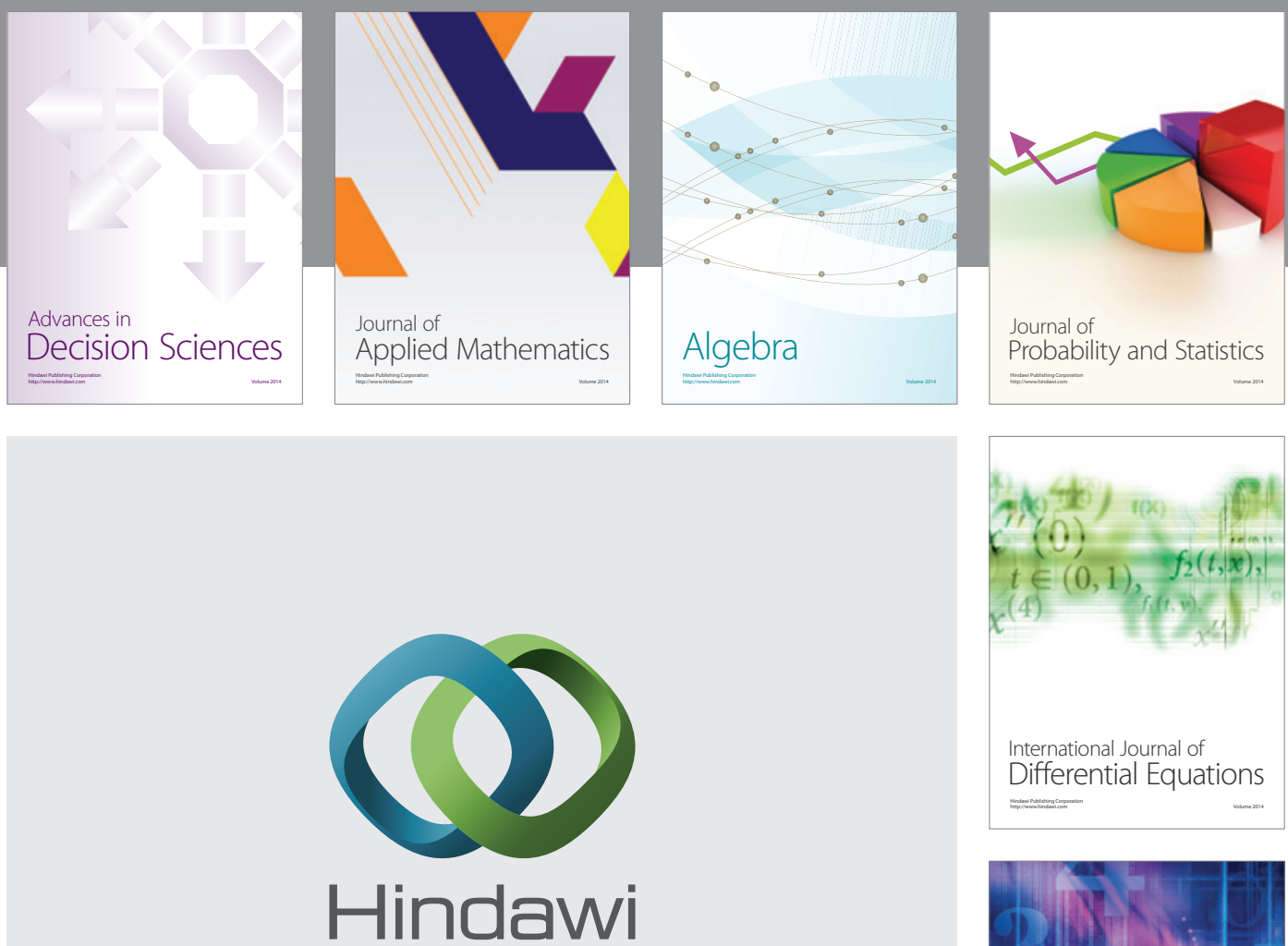

Submit your manuscripts at http://www.hindawi.com
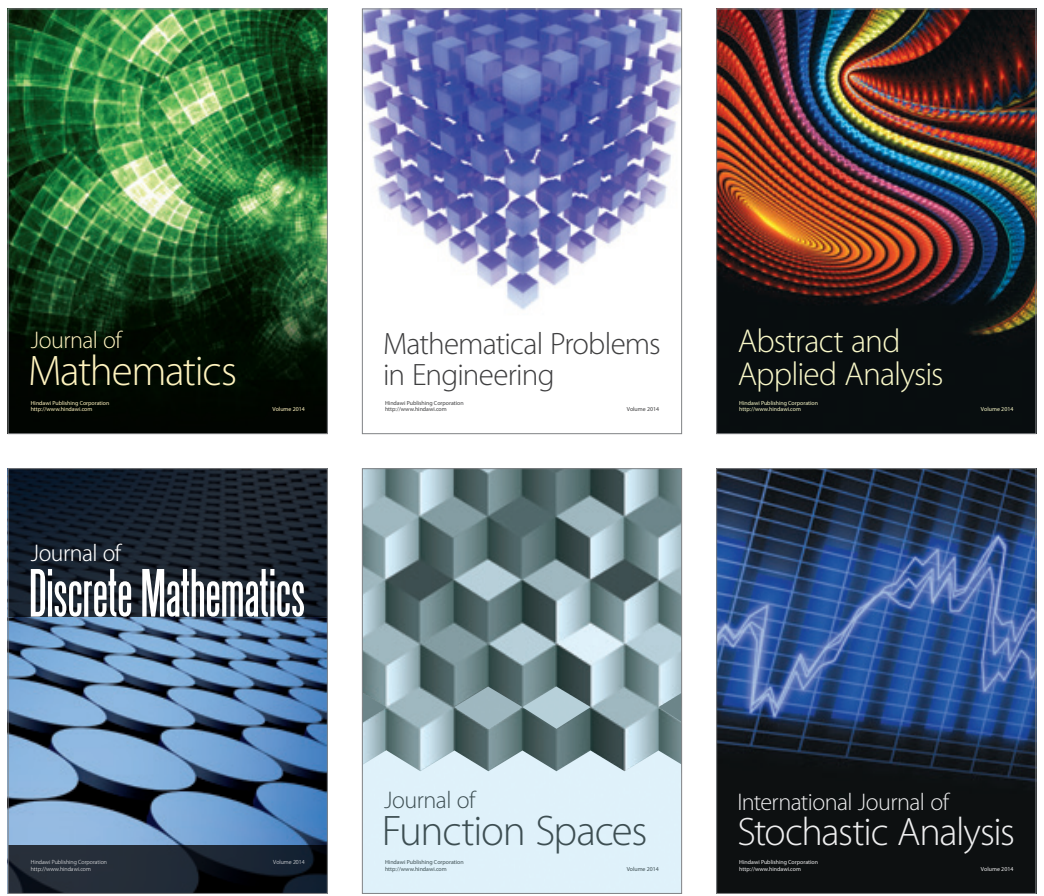

Journal of

Function Spaces

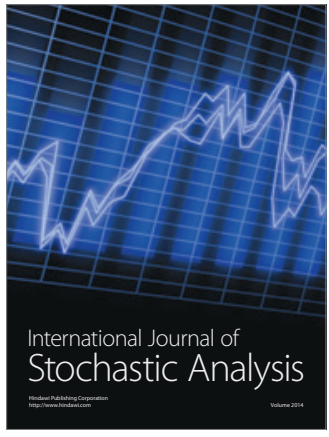

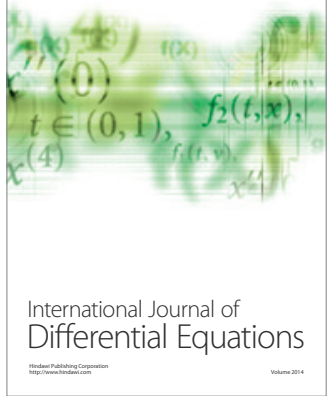
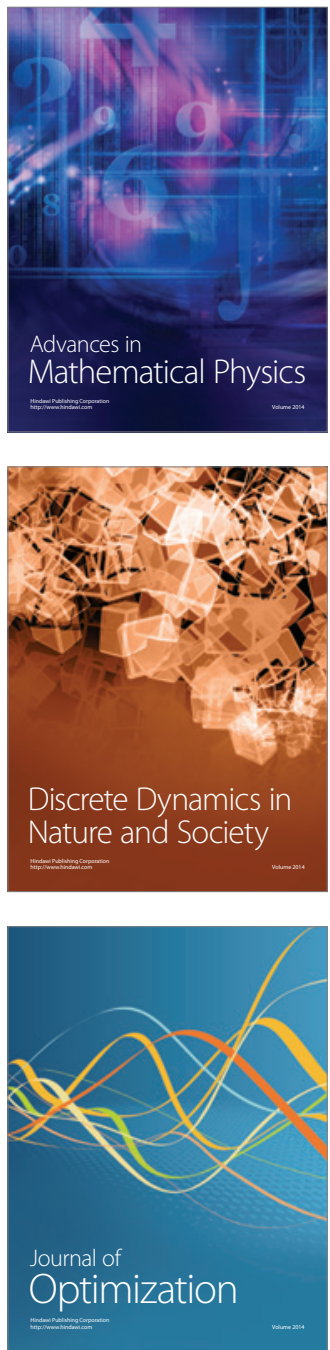